\title{
ACCURACY CALIBRATION OF THE BARS OF LEVELLING METERS
}

\author{
Vytautas Giniotis $^{1}$, Donatas Rekus ${ }^{2}$, Česlovas Aksamitauskas ${ }^{2}$ \\ ${ }^{1}$ Institute of Geodesy, ${ }^{2}$ Dept of Geodesy and Cadastre, Vilnius Gediminas Technical University, \\ Sauletekio al. 11, LT-10223 Vilnius, Lithuania \\ E-mail: gkk@ap.vgtu.lt
}

Received 3006 2007, accepted 27092007

\begin{abstract}
Precision levelling is one of the most common geodetic measuring operations. The most modern geodetic instruments are quite sophisticated digital instruments that are supplied with photoelectric sensor arrays and opticalelectronic cameras that fix the height values of terrain according to the position of the levelling meter that is placed on it. The marks on the levelling meters are also modified and consist of coded strokes put on the meter's surface in a specific order. The accuracy of the performance of all measuring equipment depends mostly on the accuracy of the position of those strokes. This paper deals with an analysis of an accuracy calibration of levelling meters, its methods and means of evaluation.
\end{abstract}

Keywords: levelling instrument, meter, accuracy, errors, calibration, measurement.

\section{Introduction}

The operation of levelling instruments is based on the digital processing of an image of the coded meter. Every company producing levelling instruments has developed its own codes and methods of processing: correlation (Leica Wild NA2000/2002/3000/3003 systems), geometric method (Zeiss DiNi 10/20 systems), and phase method (Topcon DL - 101/102 systems) [1-4].

At the beginning of measurement, a visual pointing of the instrument onto the surface of the levelling meter is performed. After that, the instrument automatically points the focus of its optical system on the surface of the meter and then a rough correlation calculation is performed followed by the precise correlation. About 50000 correlation calculations are performed for full parameter determinations. According to data received in the processor of the instrument, an exact distance from the axes of the instrument to the surface of the level meter is calculated. According to the information received by decoding it from the photoelectric matrix, the height of the level placing is calculated in the processor. During this operation the coded view of the meter is compared with that saved in the instrument memory. A true meter's height position is determined according to the shift of the image in the photoelectric sensor (pixels) matrix.

A $30 \mathrm{~cm}$ part of the meter is enough for the determination of the height and distance in the DiNi $10 / 20$ system. The levelling meter is divided into strokes of $2 \mathrm{~cm}$ length; while other systems use $3 \mathrm{~cm}$ length strokes. A phase shift is ensured between the two types of strokes along the full length of the meter. The third type of strokes is used for giving additional frequency information, and all these systems are used for the determining of the height and the distance. Processing of information is quite complicated in various levelling systems. For example, in Leica systems the code bars (strokes) are put on the full length $-4,050 \mathrm{~mm}$ of the meter. The bar code has 2,000 elements, each with a width of $2,025 \mathrm{~mm}$. It is evident that the production of such a meter is quite complicated; its accuracy mostly depends on the errors of placement of the coded bars. The matrix of the photodiodes (pixels) has 256 elements that are placed with the pitch of $25 \mu \mathrm{m}$ from each other. Accuracy of the placement of these cells also influences the overall accuracy of the instrument's performance.

Accuracy of the calibration of placement of the coded bars on the surface of meters is analysed in this paper.

\section{Accuracy calibration of the meters}

All coded meters can be calibrated by interferometric comparators. Usually, it is enough to measure every stroke (or bar) of 2,025 mm width at the determined pitch. The gaps between the bars are at distances in times of 2,025 mm. Vertical comparators are mostly used for this purpose. By applying horizontal comparators the thermal expansion coefficients and thermal resistance of a meter are investigated. The meters are calibrated at $0,10,20,30$ and $40{ }^{\circ} \mathrm{C}$.

The comparators partly repeat the operations of levelling. Some of the height levelling can be used for measurement or calibration of precision levelling meters. Usually the vertical position of the calibration is used for keeping the same position as it is during the work operations. Nevertheless, there are variants of calibration in the horizontal position, as it saves the height of the 
calibration laboratory and makes an operation easier. The vertical comparators are arranged at Graz University of Technology (Austria), at the Institute of Geodesy (Finland), and Munich Bundeswehr University (Germany). The horizontal structure of the comparator is applied at ETH University in Zurich. The main technical features of the comparators are given in Table 1.

Table 1. Principal structure of comparators

\begin{tabular}{|l|l|l|}
\hline Placement & \multicolumn{1}{|c|}{ Standard used } & \multicolumn{1}{c|}{ Moving part } \\
\hline Vertical & Etalon meter & Calibrated meter \\
\hline Horizontal & Laser interferometer & $\begin{array}{l}\text { Carriage with CCD } \\
\text { camera }\end{array}$ \\
\hline & $\begin{array}{l}\text { Photogrammetric view } \\
\text { of standard meter }\end{array}$ & $\begin{array}{l}\text { Carriage with CCD } \\
\text { camera and reflec- } \\
\text { ting mirror }\end{array}$ \\
\hline & & $\begin{array}{l}\text { Without mutual } \\
\text { movement }\end{array}$ \\
\hline
\end{tabular}

The main operational principle of the comparator is as follows: the meter to be calibrated is lifted by means of the step motor and special lifting arrangements. The position of the meter is read by the laser interferometer and the edges of the meter"s bars are fixed by the CCD camera. An automatic climate control system registers an ambient temperature, pressure, and humidity, as the length of the meter and laser light propagation parameters must be corrected in accordance with environmental parameters. All this system is controlled by the PC.

Vertical and horizontal comparators consist of complicated maintenance and construction, they are quite expensive and large in size. The laboratory room must be high for the vertical comparator and long - for the horizontal comparator. Besides, an expensive measuring equipment is used - the laser interferometer.

\section{Photogrammetric calibrations}

The principles of photogrammetry can be used for comparing the views of the standard and meter to be calibrated. The correlation factor determination can be used successfully as well, as in the case of a Digital levelling operation. The standard view is saved in the PC memory, and the view of the tested meter must be extracted from the CCD camera. All measurements are performed on the PC screen by an analysing gap covering both images (Figs 1, 2).

At first, adjustments of the meters' views in the direction of $x$ and $y$ axes must be taken. As it is seen in Fig 1, a bad image projection causes the faults in the images, a non-horizontal alignment - in the cosine error to be present at the misalignment angle $\vartheta$. It is evident that the true length $h$ of the image will differ from the misaligned length $l$ as $\Delta l=l(1-l \cos \vartheta)$.

There is an opportunity to shift the meters along each other on the computer screen. The CCD camera is supplied by the analysing window for selecting the part of the meters that are chosen for the comparison between themselves [5-9]. The analysing window is shifted along the length of the meters or moved by steps at least three times - at the beginning, in the middle and at the end of metering. In this case, it is easier to apply a computer zooming process for the part to be compared (Fig 1).

Preliminary readings from the calibrated meter are shown in Table 2. They are taken by the standard software AutoCad. They are shown only as an example without any statistical processing. According to the example, it can be stated that the accuracy of measurement by using this method can reach tens of microns on average.

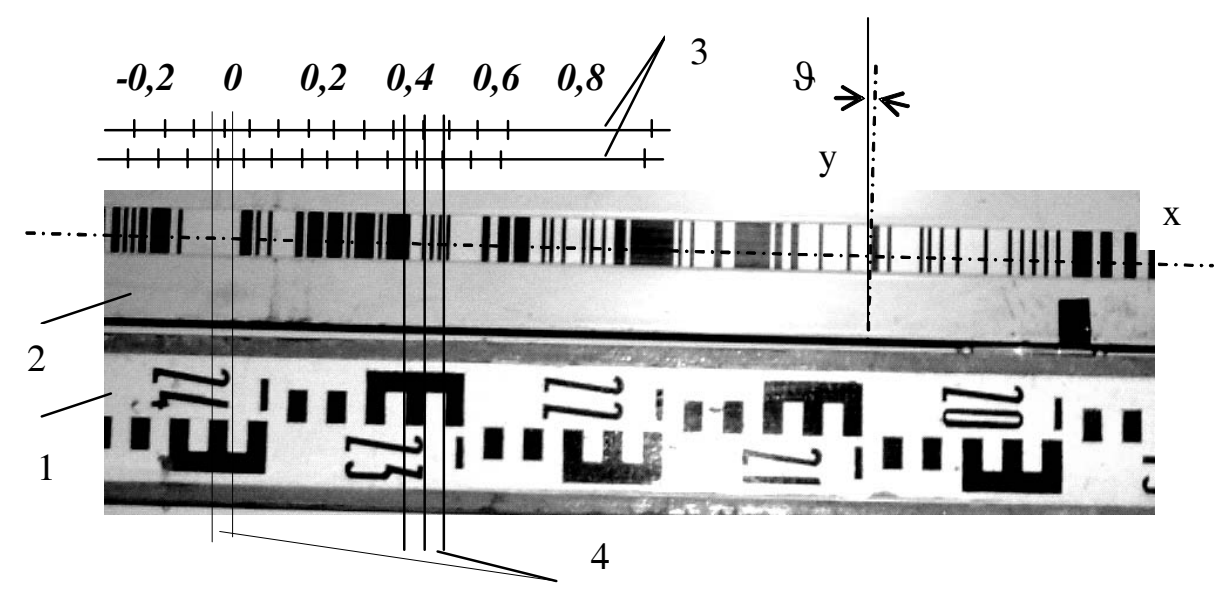

Fig 1. General view of photogrametric calibration of levelling meters: 1 - standard meter, 2 - meter to be calibrated, 3 - linear and vernier scale for readings, 4 - moving reticle 
Table 2. Example of readings from the meter

\begin{tabular}{|c|c|c|c|c|c|c|}
\hline No & $\begin{array}{c}\text { Interval, } \\
\Delta, \mathrm{mm}\end{array}$ & $\begin{array}{c}\text { Readings, } \\
\mathrm{mm}\end{array}$ & $\begin{array}{c}\text { Interval, } \\
\Delta, \mathrm{mm}\end{array}$ & $\begin{array}{c}\text { Readings, } \\
\mathrm{mm}\end{array}$ & $\begin{array}{c}\text { Interval, } \\
\Delta, \mathrm{mm}\end{array}$ & $\begin{array}{c}\text { Readings, } \\
\mathrm{mm}\end{array}$ \\
\hline 1 & 0,5 & 0,59 & 0,6 & 0,63 & 0,7 & 0.73 \\
\hline 2 & & 0,54 & & 0,60 & & 0,69 \\
\hline 3 & & 0,49 & & 0,55 & & 0,65 \\
\hline 4 & & 0,49 & & 0,55 & & 0,69 \\
\hline 5 & & & & 0,59 & & 0,73 \\
\hline
\end{tabular}

An accurate comparison of the meters can be accomplished by applying the correlation method. A better case to apply this, is a comparison of the same type of meters, for example, both coded meters by the same producer. The signals from the photoelectric sensors (pixels) are output to the PC making an array of numerical values from both meters at the same position. This coincides with the known area-based method described in [10]. The difference is only the aim of its application. In our case, it is the comparison of the position of the edges of the bars on the tested meter. The processing of the images is completed in the same way as in the method above. Smaller subarrays from both arrays are taken and the correlation factor is calculated by the formula [10]:

$$
r=\frac{\sum_{i=1}^{m} \sum_{j=1}^{n}\left[\left(A_{i j}-\bar{A}\right)\left(B_{i j}-\bar{B}\right)\right]}{\left\{\left[\sum_{i=1}^{m} \sum_{j=1}^{n}\left(A_{i j}-\bar{A}\right)^{2}\right]\left[\sum_{i=1}^{m} \sum_{j=1}^{n}\left(B_{i j}-\bar{B}\right)^{2}\right]\right\}^{1 / 2}},(1)
$$

$r$ - correlation coefficient; $m$ and $n$ - numbers of rows and columns; $A_{i j}$ - digital number from subarray $A$ in the row $i$ and in the column $j ; \bar{A}$ - average of all digital numbers in subarray $A$, and $\bar{B}$ - average of all digital numbers in subarray $B . i=0,1,2,3, \ldots, m ; j=0,1,2$, $3, \ldots, n$.

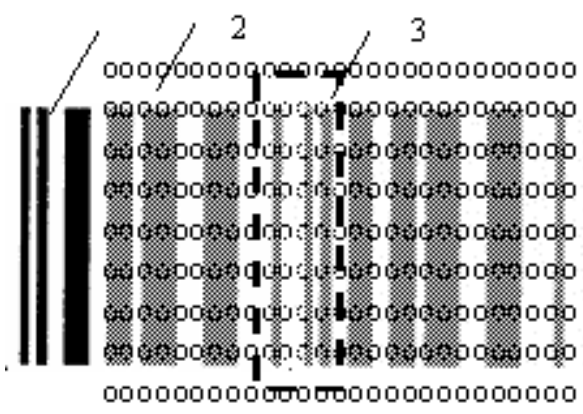

000000000000000000000000000

Fig 2. Array of photosensors and bars of meter on it: 1 - coded bars of meter, 2 - array of CCD sensors, 3 - analysing window

It is evident that a total array of numbers representing the position of the bars on the surface of the levels will be received from the array of sensors of the CCD camera, converting voltage output into the digital form. General diagram of photosensors (pixels) with the view of the coded bar of levelling meter and the analysing window are shown in Fig 2.

$$
\begin{aligned}
\boldsymbol{A} & =\left[\begin{array}{ccccc}
a_{11} & a_{12} & a_{13} & a_{14} & \ldots \\
a_{21} & a_{22} & a_{23} & a_{24} & \ldots \\
a_{31} & a_{32} & a_{33} & a_{34} & \ldots \\
a_{41} & a_{42} & a_{43} & a_{44} & \ldots \\
a_{51} & a_{52} & a_{53} & a_{54} & \ldots \\
\ldots & \ldots & \ldots & \ldots & \ldots
\end{array}\right] \\
\boldsymbol{B} & =\left[\begin{array}{c:cccc}
b_{11} & b_{12} & b_{13} & b_{14} & \ldots \\
b_{21} & b_{22} & b_{23} & b_{24} & \ldots \\
b_{31} & b_{32} & b_{33} & b_{34} & \ldots \\
b_{41} & b_{42} & \overline{b_{43}} & b_{44} & \ldots \\
b_{51} & b_{52} & b_{53} & b_{54} & \ldots \\
\ldots & \ldots & \ldots & \ldots & \ldots
\end{array}\right]
\end{aligned}
$$

Fig 3. Digital output from search array of photosensors and analyzing window for subarrays selection

A digital output from the photosensors presented in the form of matrixes $\boldsymbol{A}$ and $\boldsymbol{B}$, from the view of etalon meter and calibrated meter respectively, are shown in Fig 3.

The correlation coefficient is calculated from the subarrays that are selected by the analysing window. The error of the displacement (differences between the positions of bars on the meters) is determined by the local correlation coefficient. Such operations are performed as follows:

- Preliminary correlation coefficient calculation at the beginning, middle and the end part of the meters using the formula (1);

- $\quad$ Shift of the subarray $\boldsymbol{B}$ at the predetermined pitch $\Delta_{t}$ and repeating an operation as described above;

- Repeating the first two operations in the investigation region $\pm k \Delta_{t}$, where $k=1,2,3, \ldots$ covering the zone of expected error of the meter's bars placement;

- Statistical processing of the results of correlation coefficient calculations determining the position in the discrete numbers of pitches $\Delta_{\mathrm{t}}$ in the length $x$ of the meter.

A more exact evaluation of the bar edges can be performed by the feature-based digital image assessment technique. In case of a blurred image of the meters, additional statistical means must be taken for determining the position of the bars. The values of the photogrammetric points of the image are assessed by evaluating the standard deviations $S_{x}, S_{y}$ and 


$$
S_{x y}=\sum(x, y)-\frac{\left(\sum x_{i}\right)\left(\sum y_{i}\right)}{n} .
$$

Both coordinates $x$ and $y$ are needed for better determining the position of the bar's points (Fig 1). Linear regression equation $y=\alpha+\beta x$ can be used for this as well, where $\beta=\frac{d x}{d y}$ is the slope of the line, and parameter $\alpha$ is a constant at value $x=0$. The same can be determined as $\beta=\frac{S_{x y}}{S_{x}^{2}} ; \quad \alpha=\frac{\sum y_{i}}{n}-\beta \frac{\sum x_{i}}{n}$.

Accordingly, the sample correlation coefficient can be calculated as:

$$
r=\frac{S_{x y}}{\sqrt{S_{x}^{2} S_{y}^{2}}} .
$$

Such calculations, as presented in [10], would be easier for determining the points on the meter's bar edges, and their systematic error will be found by changing the pitch $\Delta_{\mathrm{t}}$ of a subarray sample selection.

By using the analysing window on the search array of digital numbers, its position must be changed by steps at the chosen pitch $\Delta_{\mathrm{t}}$. Therefore, maximal value of correlation coefficients will be determined at some values $i \Delta_{\mathrm{t}}$ of the meter's length, different from those on the standard meter. This difference will be equal to the systematic error of the bars position along the whole length.

\section{Conclusions}

Preliminary experiments show a possibility to perform the calibration of levelling meters by photogrammetric methods of its comparison. Digital output array processing by an analysing window is to be used. An area based comparison method applied for general purpose photogrammetry can be introduced for calculating the correlation coefficients at the shift of the images to each other by the discrete pitch that show the difference between the positions of meter's bar edges where maximal values of the correlation coefficient exist. The systematic error of the meter under the calibration is determined by the values of position where these maximal values are found along the total length of the meter.

\section{References}

1. INGENSAND, H. The evolution of digital levelling techniques - limitations and new solutions. In Geodesy Surveying in the Future. The Importance of Heights. Proceedings, Gavle, Sweden, 1999, p. 59-68.

2. NA 2002. NA 3003. Technical report, digital levels. Leica Geosystems AG, Geodesy, CH-9435 Heerbrugg (Switzerland), 1997.
3. MENZEL, M. The development of levels during the past 25 years, with special emphasis on the NI002 optical geodetic level and the $\mathrm{DiNi}^{\circledR} 11$ digital level. In Geodesy and surveying in the future. The importance of heights. Proceedings, 1999, p. 85-93.

4. INGENSAND, H.; MEISSL, A. Digital Levels on the Way to 3-D Measurements. In Conference on Optical 3-D Measurement Techniques, Technische Universität Wien, 1995.

5. SKEIVALAS, J. An accuracy of determination of the covariation of random values. Geodesy and Cartography (Geodezija ir kartografija), 1999, Vol XXV, No 4, p. 156158 (in Lithuanian).

6. GRATTAN, K. T. V.; SKEIVALAS J. and GINIOTIS, V. Development of 2D optical measurements. In Proceedings of XVII IMEKO World Congress, IMEKO and HMD, 2003, p. 1831-1833.

7. GINIOTIS, V.; SKEIVALAS, J. The level. Lithuanian Patent LT 4966 B, Int. Cl. G01C 5/02 / Vilnius Gediminas Technical University, Vilnius: State Patent Office, 2002. $10 \mathrm{p}$.

8. LOPEZ-ALONSO, M.; ALDA, J. Operational parametrization of the $1 / \mathrm{f}$ noise of a sequence of frames by means of the principal component analysis in focal plane arrays. Opt. Eng., 2003, 42(7), p. 1915-1922.

9. TAKALO, M. and ROUHIAINEN. On use of FGI system calibration comparator. Finnish Geodetic Institute, Paris, France, 2003.

10. Manual of photogrammetry. Fifth edition. Editor J. Chris McGlone, American Society for and Remote sensing.

Vytautas GINIOTIS. Prof, Doctor Habil. Vilnius Gediminas Technical University. Institute of Geodesy. Sauletekio al. 11, LT-10223 Vilnius, Lithuania. Ph +370 52744 703, Fax +370 52744 705, e-mail: vg@ai.vgtu.lt

Author of one and co-author of many other monographs and more than 160 scientific papers. Participated in many intern conferences, research and lecture visits to Germany, Austria and UK universities.

Research interests: 3D measurements and instrumentation, automation of measurements, angular measurements.

Donatas REKUS. Doctoral student. Vilnius Gediminas Technical University. Dept of Geodesy and Cadastre. Saulètekio al. 11, LT-10223 Vilnius, Lithuania. Ph +370 5 2744705, Fax +370 5 2744705, e-mail: gkk@ap.vgtu.lt.

A graduate of Vilnius Gediminas Technical University (VGTU) (MSc, 2005).

Research interests: geodetic networks, calibration of geodetic instruments.

Česlovas AKSAMITAUSKAS. Assoc Prof, Doctor. Vilnius Gediminas Technical University. Dept of Geodesy and Cadastre. Saulètekio al. 11, LT-10223 Vilnius, Lithuania. $\mathrm{Ph}+37052744703$, Fax +370 52744705 , e-mail: gkk@ap.vgtu.lt.

Doctor (1990). Author of two teaching books and more than 15 scientific papers. Participated in many intern conferences.

Research interests: geodetic instrumentation, automation of measurements, angular and distance measurements. 